\title{
Sistemas de información geográfica
}

A lejandro Flameno

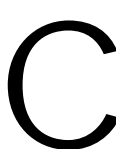

omo seres que nos desenvolvemos en un ambiente multidimensional, estamos acostumbrados a pensar con referencia a la dimensión espacial. De esta manera, el arriba, a un lado o enfrente son conceptos que nos acompañan siempre y que comprendemos de inmediato. Ubicar en dónde se encuentra cierta persona o si determinada dirección postal está cerca o lejos, son tareas que hacemos casi sin meditar en las implicaciones de ubicación quellevan consigo. Las cosas se complican un poco cuando tenemos que revisar un croquis de ubicación en una plaza comercial o en una zona arqueológica; a algunos se nos dificulta encontrar la indicación de "usted está aquí". Cuántas veces no nos ha pasado que queremos saber en dónde está el norte o buscamos el bosquecito que nos recomendaron, al sur del Huitepec, y extrañamos la brújula que venía en la bolsita de Sonric's de nuestro sobrino.

Todo esto viene a colación porque no se me ocurría cómo empezar a hablar del tema de los sistemas de información geográfica (SIG). Q ué son, cómo se estructuran, sus alcances, limitaciones u operación son temas relativamente sencillos de explicar, sin embargo, hay dos puntos sobre los que se me complica escribir: "por qué existen" y "para qué sirven", pues en realidad ambos nos parecen obvios a quienes trabajamos en el Laboratorio de Análisis de Información Geográfica y Estadística (LAIGE), debido a nuestro trato continuo con el tema, aunque seguramente no es así para el resto de la gente que no tiene que perder su tiempo usando los SIG.

Podría empezar, como muchos libros al respecto, diciendo que un SIG es un conjunto de equipos y programas de cómputo utilizados para almacenar, manipular y analizar datos espacialmente referenciados, ¿de qué les sirve saber esto a los lectores? Sería como describir una videocasetera o un reproductor de compactos. Q uienes hemos lidiado con los fierros y los programas sabemos que estas "cosas" no son realmente lo que constituye la parte importante de un SIG, pues son el compo19. nente humano y el tipo de información que se maneja la materia primordial de estos sistemas, pero antes de entrar en descripciones y comentarios quiero discutir sobre su razón, que en buena medida es ayudar a entender nuestro entorno. Al princiio hablaba de nuestra capacidad de concebir el mundo dentro de una dimensión espacial. En adelante, cuando use éste término me referiré al espacio que es parte de la superficie terrestre, incluyendo su totalidad, y no al espacio exterior.

Casi cualquier disciplina que se nos ocurra trata con fenómenos u objetos que podemos, con mayor 0 menor facilidad, ubicar en la dimensión espacial. En muchos casos, los objetos de estudio necesitan ser aproximados no sólo con base en su ubicación geográfica, sino respecto a la relación que mantienen con su entorno (Cambrezy, 1997). Hay dos actividades en que los SIG apoyan los estudios relacionados con la realidad: la descripción y el análisis.

\section{La descripción de los fenómenos}

La descripción de un objeto o fenómeno es más completa si se considera su ubicación y las relaciones que mantiene con su entorno, es decir, su contexto. Un problema común es la delimitación de dichos objetos. Podemos pensar en líneas divisorias, pero la naturaleza rara vez presenta tal tipo de límites. Esto implica dos problemas, pues por un lado las líneas finas son la excepción, 
pero por otra parte no se sabe mucho acerca de los límites, aunque a medida que aumenta el conocimiento, éstos se definen con mayor precisión a pesar de seguir manteniendo una naturaleza difusa.

Muchos de los objetos de estudio de disciplinas como la ecología pueden ser concebidos sin recurrir a su dimensión espacial. El ecosistema es un concepto y por tanto no tiene un espacio definido, pero si consideramos al paisaje como su expresión espacial, entonces el espacio se convierte en un atributo relevante. D e la misma manera, la marginación, las actividades económicas, la influencia de los medios de comunicación o un proceso de especiación pueden encontrar ventanas descriptivas a través de su expresión en el espacio.

Buena parte de los trabajos de descripción se llevan a cabo sin necesidad de un SIG; en ocasiones basta contar con un mapa para describir algún aspecto en particular. Un mapa es un medio analógico compuesto por líneas, colores y símbolos que se analizan y crean de manera manual (Berry, 1993). Suelen estar impresos en papel 0 algún otro material y cumplen con una serie de características que los hacen diferentes de cualquier otra figura impresa. Si una imagen puede decir más que mil palabras, podemos entonces ponderar la capacidad descriptiva de un mapa para una inmensa variedad de temas.

La interpretación de los mapas de una manera visual y manual - utilizando este término como lo opuesto a automatizado- fue durante muchos años el procedimiento común para la interpretación y análisis de información geográfica. El proceso del manejo de la información espacial ha seguido un continuo en que se ha aprovechado el desarrollo de nuevas tecnologías.

Un SIG es un conjunto de elementos de distinta naturaleza cuyo objetivo es llevar a cabo tareas de acopio, almacenamiento, manipulación, análisis, medición, transformación y representación de información que tiene una referencia espacial o geográfica. Permite añadir la dimensión temporal, ya sea por la naturaleza de los datos, su capacidad de actualización y algunos procesos de análisis, como las series de tiempo.

En este terreno es importante entender los conceptos de escala y resolución. La escala es una relación entre un valor del modelo (en este caso el mapa) y un valor de la realidad. Si en un mapa midiéramos una mancha que representa una cancha de fútbol, su tamaño va a ser una fracción de lo que la cancha mide en

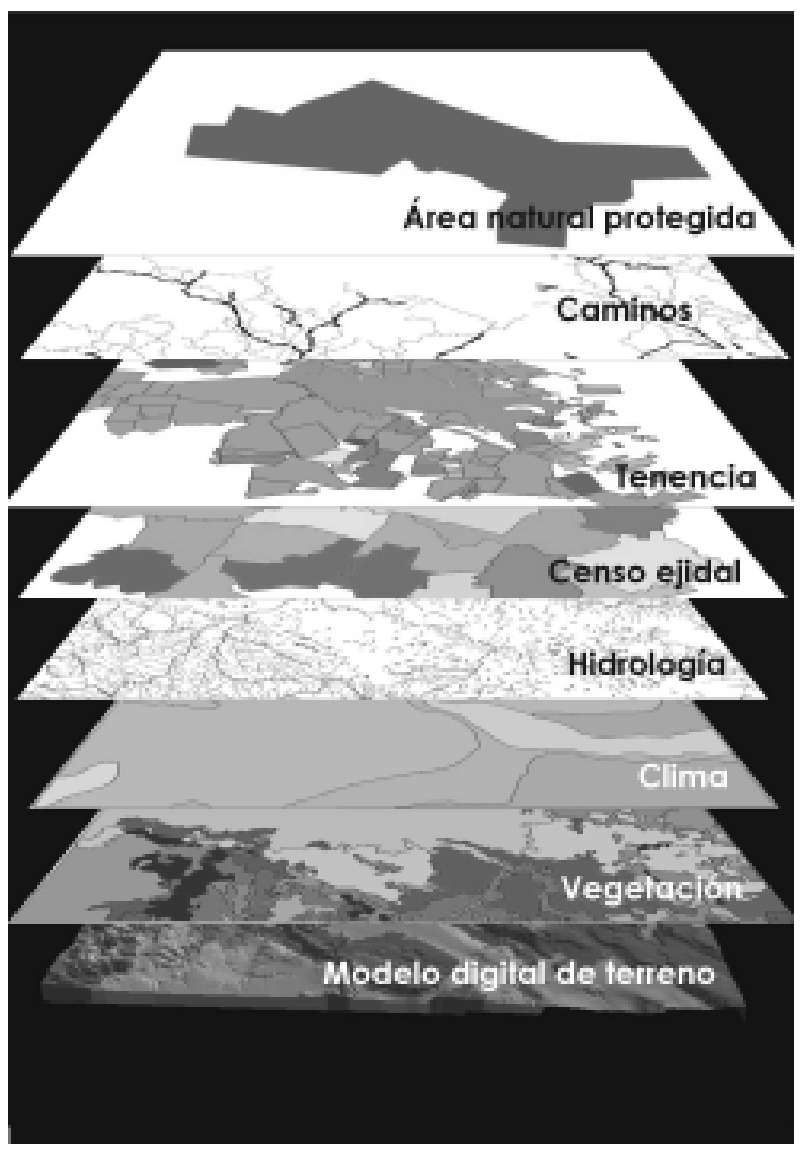

realidad. Si ésta midiera 10 centímetros de largo en un mapa escala 1:1,000 (que se lee uno a mil) significaría que en la realidad mediría 100 metros pues cada unidad en el mapa representa mil unidades reales $(0.10 \mathrm{x}$ $1,000=100$ ).

La resolución se refiere al proceso o capacidad de hacer distinguibles las partes individuales de un objeto. Para quienes han tenido que elegir monitor de computadora sabrán que es mejor tener una resolución de 1024 x 768 que uno de 800 x 600 pixeles. La resolución es la relación que existe entre el modelo con la realidad. Cuando se elabora un mapa se lleva a cabo una estratificación de la realidad que corresponde con el tema de interés de quien lo realiza y del público al que va dirigido. Esto es cierto para cualquier tipo de información y en parte se debe a la imposibilidad de representar toda la realidad. Q uien extrae la información tiene que discriminar los objetos de los que requiere obtener datos, el tipo de datos que necesita y establecer un método de clasificación que le permita organizarlos. Alguien que 
lleva a cabo una investigación de mercado tendría que tomar decisiones sobre los grupos de edad que necesita evaluar y los intervalos de edades que comprendería cada grupo.

Cuando se interpreta la información espacial, ésta se puede desmenuzar 0 agrupar de acuerdo a distintas necesidades. Al revisar una carta topográfica de 1:50,000 de las que produce el Instituto Nacional de Estadística, G eografía e Informática (INEGI), es posible encontrar simbolizadas áreas con cobertura forestal. Uno podría pensar que esto bastaría para saber cuál es la cobertura forestal de un área, sin embargo, se incluyen sin distinción plantaciones forestales, de árboles frutales o áreas de reforestación. No podemos saber si son bosques templados o selvas ni el estado sucesional de la vegetación. Además, la intención de esas áreas en un mapa topográfico es advertir que las curvas de nivel que hay en ellas no son del todo confiables, debido al ruido que ocasiona la cobertura de árboles, así que el objetivo con que se indicaron no fue el de evaluar masas forestales.

Por otra parte, si se contara con una carta de uso del suelo y vegetación en la misma escala para la misma zona, la resolución en cuanto a los tipos de cobertura forestal sin duda sería suficiente para el propósito indicado. Éste es un ejemplo en que la escala puede ser la misma mientras que la resolución es distinta.

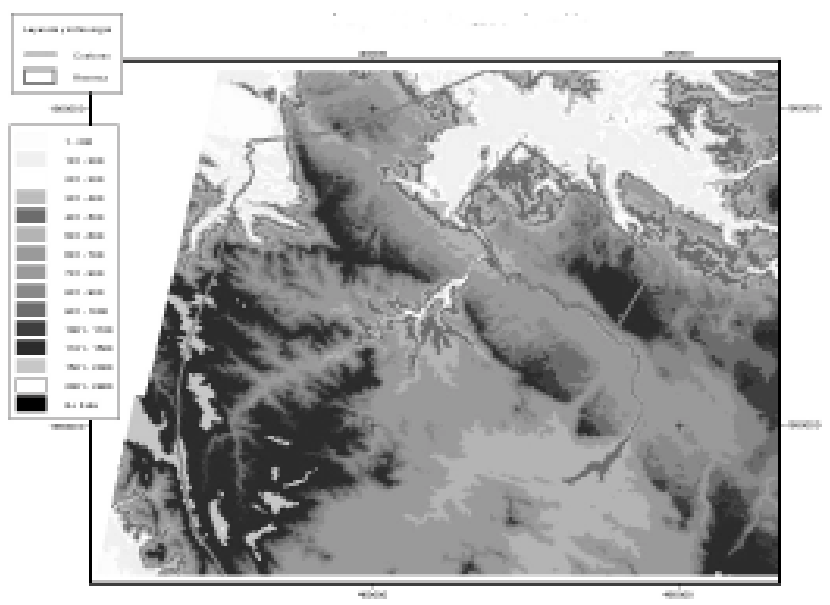

\section{El análisis espacial}

Con el desarrollo de la humanidad se ha acumulado un creciente acervo de información, que ha permitido hacer interpretaciones más precisas de la realidad y también realizar procesos de análisis de esta realidad cada vez más complejos.
El análisis de la información geográfica puede ser realizado de manera manual o automatizada (D antasBitancourt y Regina-Pivello, 1998; Berry, 1993), pero entre mayor es la cantidad de información, más complicado es realizar el análisis manual. A mediados del siglo pasado la tarea de sobreponer mapas temáticos impresos sobre acetatos transparentes era un procedimiento

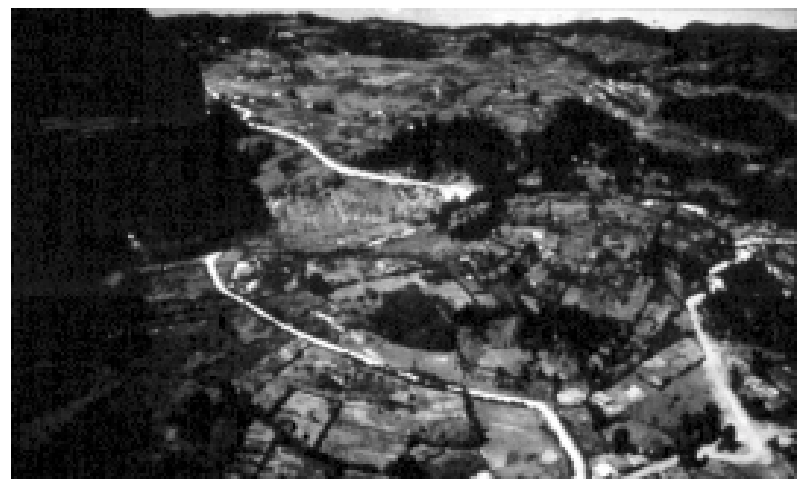

común para realizar distintos análisis. D esde luego que entre mayor cantidad de información se necesitaba analizar, el procedimiento se volvía más complicado. Por otra parte, el uso mismo de los mapas se encuentra con algunas limitaciones, la primera es conseguirlos. ¿Alguien ha buscado un mapa de los ámbitos de distribución del jaguar en Chiapas? ¿Sabe donde comprarlos? Claro que se puede inferir a partir de otras capas de información, como la relacionada con el hábitat, y de otros datos, como la proximidad a poblados o el grado de perturbación de la vegetación, aunque en muchas ocasiones tampoco se cuenta con esa información plasmada en mapas.

Otro problema serio es el tiempo que se necesita para realizar un mapa. Por ejemplo, la carta de vegetación de uso del suelo y vegetación de Tuxtla Gutiérrez fue publicada en 1984 por el INEGI, pero las fotografías aéreas en que se basa datan de 1973, 1974 y 1979. $\mathrm{Si}$ alguien toma su mapa y se dirige a un manchón de bosque mesófilo de montaña para encontrar una salamandra endémica a ese tipo de vegetación en los Altos de Chiapas y que sólo se registró en una localidad, corre el riego de que al llegar al sitio se encuentre con que está en un bosque "maizófilo" y su salamandra cambió de estatus, de endémica a extinta. Podríamos resumir que los mapas impresos limitan el análisis por su carácter estático.

El incremento de información se ha visto favorecido por el desarrollo de las tecnologías informáticas y las posibilidades de comunicación, que han permitido que el proceso de retroalimentación se acelere cada día. 
Esto es un punto muy importante de por qué el análisis espacial ha tenido que recurrir a métodos automatizados cada vez más sofisticados y a la vez más sencillos de operar. Utilizando las herramientas de un SIG, ahora es relativamente fácil determinar áreas, distancias, zona de influencia, o derivar mapas de pendientes, cuando los métodos de análisis manual requerían de un trabajo intenso y mucho tiempo para realizar tales cálculos.

Los SIG se han convertido en una herramienta casi indispensable para distintas áreas de la academia. Sin embargo, sigue siendo la tarea de quien analiza e interpreta los resultados el dar sentido y coherencia a la información que de ellos se deriva y la forma en que se lleva a cabo el análisis. Como mencioné antes, un SIG no son los programas de cómputo, aunque normalmente sí requiere de ellos, y tal vez la diferencia más importante con el resto de los paquetes que estamos acostumbrados a utilizar es que no basta leer el manual o la F1 para usarlo de manera adecuada.

El uso de los mapas tiene algunas limitaciones, la primera es conseguirlos. ¿Alguien ha buscado un mapa de los ámbitos de distribución del jaguar en Chiapas? ¿Sabe donde comprarlos? Claro que se puede inferir a partir de otras capas de información y de otros datos, como la proximidad a poblados o el grado de perturbación de la vegetación, aunque en ocasiones tampoco se cuenta con esa información en los mapas.

\section{¿Qué son los sistemas de información geográfica?}

Un SIG es un conjunto de elementos de distinta naturaleza cuyo objetivo es llevar a cabo tareas de acopio, almacenamiento, manipulación, análisis, medición, transformación y representación de información que tiene una referencia espacial o geográfica (Bocco et al., 1991, Bocc0, 1999; March, 1993). Estos elementos incluyen datos organizados bajo cierto criterio, normalmente un equipo y programas de cómputo especializados, métodos y dispositivos destinados a la publicación o representación de la información derivada y sobre todo, de personal especializado en el manejo de los distintos módulos y en la manipulación y análisis. Un aspecto sobresaliente de estos sistemas es que generalmente permiten

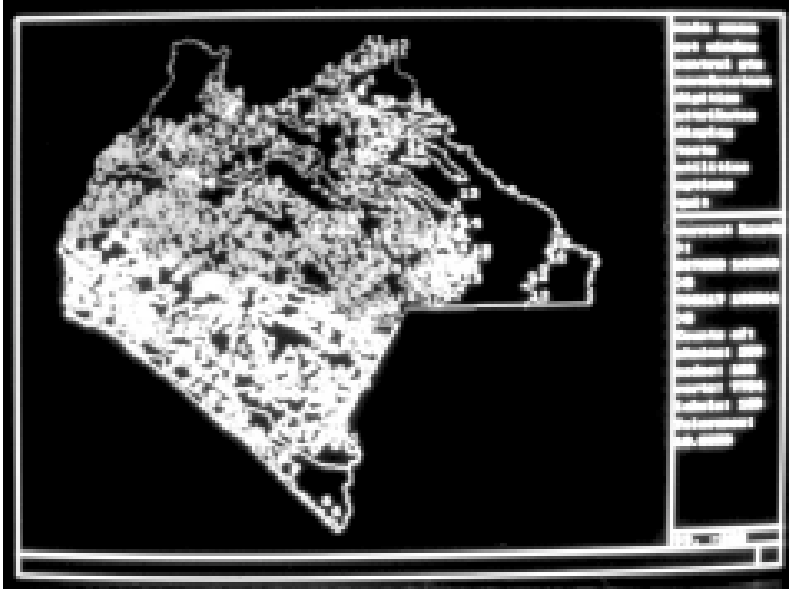

añadir la dimensión temporal, ya sea por la naturaleza de los datos, su capacidad de actualización y algunos procesos de análisis, como las series de tiempo.

Sus objetivos varían de acuerdo a sus propósitos, de tal manera que algunos están dirigidos a apoyar la toma de decisiones, otros se desarrollan con el propósito de ayudar en procesos de zonificación o planificación, algunos se orientan a tareas de monitoreo o seguimiento de ciertos rasgos, mientras que otros más buscan responder preguntas de investigación de diferentes disciplinas, tanto científicas como administrativas.

Una de las propiedades que les han dado fuerza es su versatilidad, ya que se puede usar información de diversas fuentes y en distintas formas. Además, un SIG puede generar nuevos temas u obtener información a partir de los procedimientos de análisis o consulta sobre temas existentes previamente y bajo reglas determinadas por el usuario. Regresando al ejemplo del ámbito de distribución del jaguar, quizá un científico experto en el tema no quiera dibujar un mapa al respecto, pero sí puede utilizar datos ambientales para seleccionar regiones que cumplan con las condiciones que él considera esenciales para los jaguares.

Los SIG tienen la capacidad para manejar grandes volúmenes de datos y ejecutar análisis cada vez más complejos. Los análisis de componentes principales y otros procedimientos estadísticos eran hasta hace unos años procesos poco menos que imposibles para lidiar con la información de un SIG. En la manipulación de información obtenida por métodos de percepción remota, como las imágenes de satélite, el avance de la tecnología ha abierto algunas de las ventanas más importantes para el desarrollo de información actual y precisa sobre recursos naturales. Así, los métodos de clasificación automatizada prometen hacer cada vez más eficiente el seguimiento de procesos como la de- 
forestación, cambios fisicoquímicos en las masas oceánicas o en la atmósfera.

Siempre será bueno tener en cuenta que un SIG no es una caja negra en la que metemos datos y sacamos información reluciente, bonita y que nos pone en la frontera del conocimiento de un tema. En realidad es más común de lo deseable encontrar que se mezclen peras con manzanas o se cometan pecados capitales al manejar un SIG. Uno ejemplo es cuando se manejan datos provenientes de distintas fuentes, cuya escala de origen es diferente para cada capa de información. La mayor parte de las cartas temáticas del INEG I están en escalas 1:250,000 y 1:50,000. Sería un error realizar análisis de datos procedentes de ambas y pretender que la escala de trabajo, es decir, la que se está generando, corresponde a la escala más grande. No podemos mejorar la resolución de la información de escalas más pequeñas por combinarlas con información de las mayores.

\section{Elementos de un SIG}

Un sistema de información geográfica está constituido por varios subsistemas o módulos, que a continuación describiremos: recopilación de datos, adecuación de datos, administración y manipulación de datos, análisis, representación o publicación de los resultados.

\section{Recopilación}

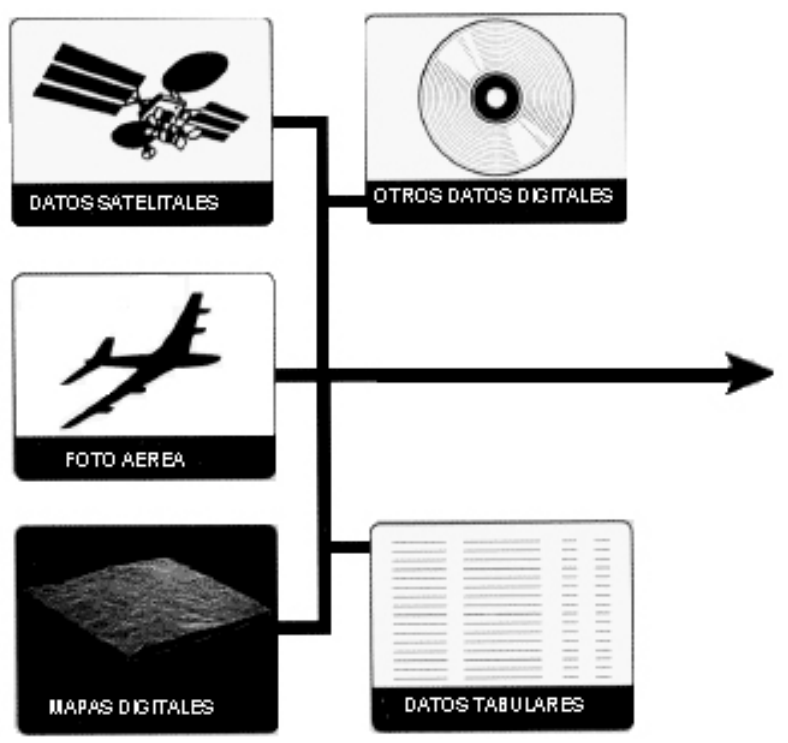

En un SIG es posible incorporar cualquier variable que pueda ubicarse espacialmente. Al hablar de variable nos referimos a los valores que puede asumir un tema determinado. La forma en que se estratifica la información depende de los requerimientos del sistema y de la disponibilidad de los datos. Así, cuando hablamos del tema o capa de información podemos pensar en temperatura media anual y las variables podrían estar indicadas en grados centígrados, que podrían además comprender fracciones de grado 0 valores enteros, dependiendo de la resolución de la información disponible. Las bases de datos de los SIG almacenan información de la ubicación de los rasgos en el espacio, pero también de los atributos de dichos rasgos.

Los SIG se han convertido en una herramienta casi indispensable para distintas áreas de la academia. Sin embargo, sigue siendo la tarea de quien analiza e interpreta los resultados el dar sentido y coherencia a la información que de ellos se deriva y la forma en que se lleva a cabo el análisis.

La ubicación de los datos puede estar determinada por coordenadas geográficas, es decir, un sistema cartesiano con referencia a la superficie de la tierra en que los ejes son referidos a la latitud (norte 0 sur) y a la longitud (este u oeste). Sin embargo, la ubicación también puede estar referida a un sistema de coordenadas relativo, que por tanto no tiene que estar referenciado al sistema de coordenadas geográficas. También es posible que se utilicen otro tipo de referencias de ubicación como el nombre de calles o códigos de área, aunque esto no es tan común.

Las fuentes de información son diversas y aunque los mapas representan un insumo primordial, existen varias otras opciones de utilidad. Muchos datos escritos pueden ser llevados a un SIG. Los datos en las etiquetas de herbario son el típico ejemplo de esta opción. También se pueden integrar otras bases de datos computarizadas. El trabajo de campo puede aportar información clave a un SIG cuando se tiene claro qué tipo de datos se requieren y de qué manera se van a sistematizar después.

El sistema de posicionamiento global - es decir mundial- (GPS por sus siglas en inglés) permite obtener la ubicación de un sitio con suficiente precisión. Está conformado por una constelación de 24 satélites que están circunnavegando la Tierra, los cuales emiten seña- 
les que son captadas por receptores que determinan su ubicación respecto a la superficie terrestre. Los receptores de este sistema son cada vez más populares y permiten, además de almacenar las coordenadas de los puntos deseados, seguir o almacenar rutas. A estos receptores generalmente les llamamos GPS.

D ebido a que en la mayoría de los casos los SIG constan de equipos de cómputo, es común que los datos se tengan que transformar a un formato digital. Cada vez es más común la posibilidad de intercambiar información en este formato y la disponibilidad de datos en sitios públicos de internet. Hoy es posible conseguir capas de información del Instituto de Geografía de la Universidad Nacional Autónoma de México o de la ConABIo, listas para integrarse a un SIG.

\section{Adecuación}

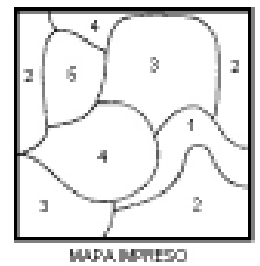

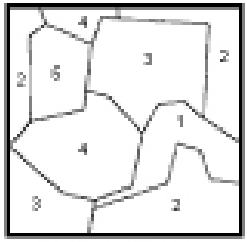

moeso vectofial

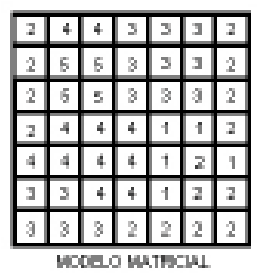

Mocedo Muthow
Una vez que se cuenta con los datos, es necesario adecuarlos a las características del SIG. Los elementos geográficos o espaciales se pueden representar con puntos, líneas o polígonos, que en realidad representan áreas. Cada uno de estos rasgos se refiere a un tipo de información, pero eso depende de los propósitos, capacidades y origen de los datos. Los asentamientos humanos se pueden señalar como puntos cuando sus datos de ubicación sólo comprenden un par de coordenadas, pero se

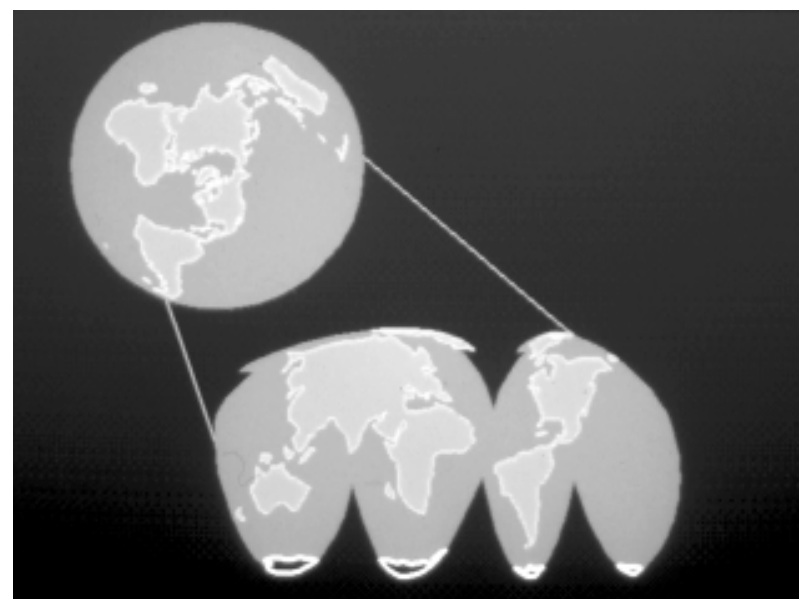

podrían plasmar con polígonos si se cuenta con la información de la mancha urbana.

En ocasiones sólo se tienen datos puntuales a partir de los cuales se generan capas basadas en interpolaciones. La información de precipitación para una serie de estaciones meteorológicas podría usarse para generar un mapa de precipitación que estaría representado por áreas.

La forma en que se incorporan los datos a un SIG se debe acoplar a un modelo determinado. Han existido varios modelos pero los preponderantes son el matricial y el vectorial. Ambos tienen ventajas y desventajas y están orientados a cumplir con determinados propósitos, aunque cada vez es más común que los paquetes de cómputo puedan utilizar ambos formatos. El modelo utilizado dependerá del tipo de información con que se cuente 0 que se requiera transformar. Un mapa de caminos podrá estar almacenado en un formato vectorial, pero la capa de precipitación interpolada a partir de estaciones climáticas debe estar en formato matricial.

Normalmente, al transferir información al SIG se deben recodificar los valores de atributo, y en ocasiones es necesario sacrificar precisión para obtener compatibilidad entre los datos. Este tratamiento de la información es crucial para el buen funcionamiento del sistema.

\section{Administración y manipulación}

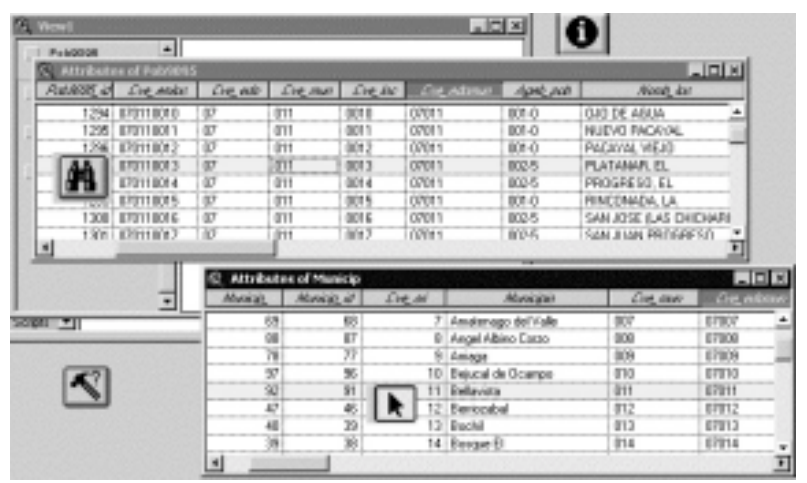

Por lo general, la información se almacena en bases de datos. Esto permite realizar en ellos actualizaciones, modificaciones o alteraciones de una manera sencilla. La versatilidad de los SIG se debe en parte a que los datos pueden compatibilizarse. O tra función de este subsistema es que permite el intercambio de información con otros SIG y así se facilita el flujo pero también el incremento de información disponible, lo que redunda en generación de nueva información más robusta, complementaria y confiable. 


\section{Análisis}

Ningún SIG puede preciarse de serlo si no cuenta con un subsistema orientado a realizar análisis, pues sería simplemente un sistema cartográfico sistematizado. Se pueden distinguir cuatro tipos generales de análisis: los de consulta, los de sobreposición, los de modelación topológica y los de redes.

En el caso de los análisis de consulta, existen dos opciones: las que se hacen a partir de la tabla de atributos y las que se hacen a partir de un tema. Ejemplo del primer caso es la selección de los registros con suelos tipo feozem (figura 1), y en el segundo caso la selección podría hacerse para los asentamientos ubicados en un radio de $5 \mathrm{~km}$ alrededor de San Cristóbal de Las Casas (figura 2). Estos análisis son de los más básicos para los SIG y en ocasiones se usan para realizar procedimientos que corresponden más bien a la manipulación de los datos.

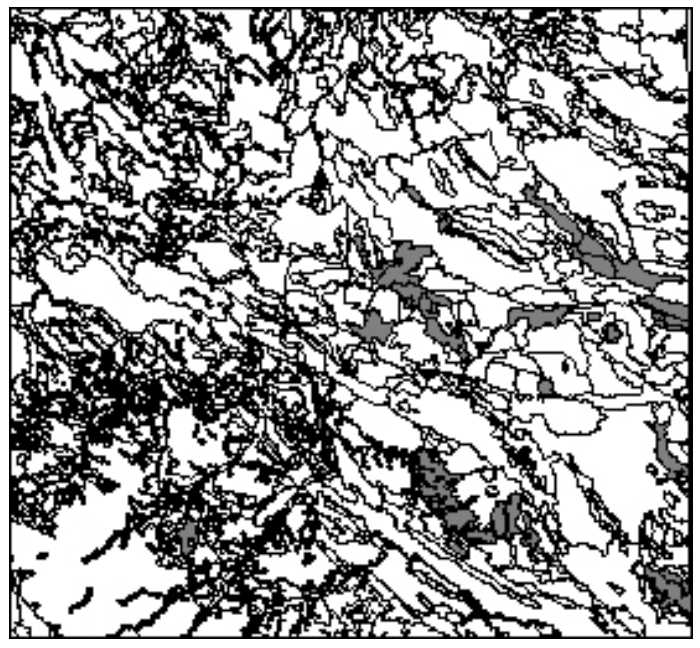

Figura 1. Los polígonos sombreados representan suelos tipo feozem.

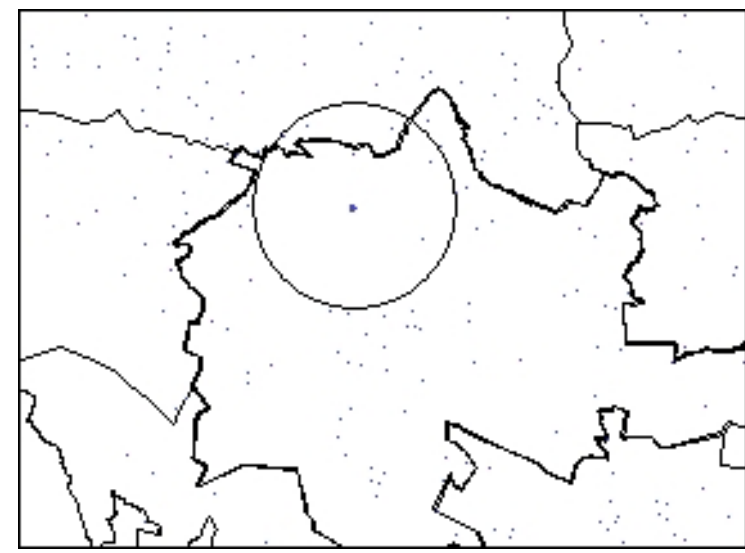

Figura 2. Localidades ubicadas a $5 \mathrm{~km}$ alrededor de San Cristóbal de Las Casas.
El análisis por sobreposición de capas es uno de los más antiguos y se llevaba a cabo desde antes de que se contara con sistemas automatizados. Se trata de encontrar concordancias, coincidencias o discordancias para áreas específicas respecto a diferentes capas de información. Con este tipo de análisis se han realizado estudios de aptitud de los suelos, evaluaciones militares, ordenamientos urbanos e incluso la elección de áreas para campos de golf.

Por otra parte, la modelación topológica se refiere a la capacidad de un SIG de reconocer y analizar la relación espacial entre rasgos. Se pueden analizar las condiciones de adyacencia, que responden a la pregunta ¿qué está cerca de qué? (figura 3); de continencia, que responde a ¿qué es contenido por qué?(figura 4), y de proximidad o ¿qué tan cerca está cierto rasgo respecto al otro? (figura 5).

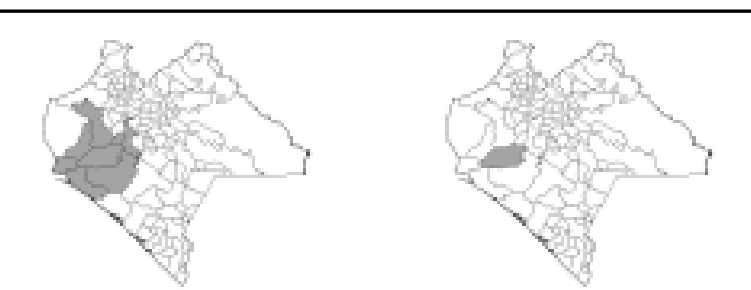

Figura 3. Ejemplo de selección por adyacencia. Se seleccionaron todos los municipios adyacentes a Villaflores.

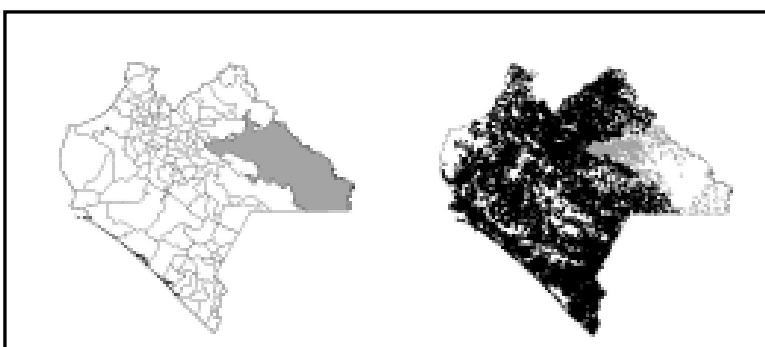

Figura 4. Ejemplo de selección por continencia. Se seleccionaron las localidades que están comprendidas dentro del polígono del municipio de 0 cosingo.

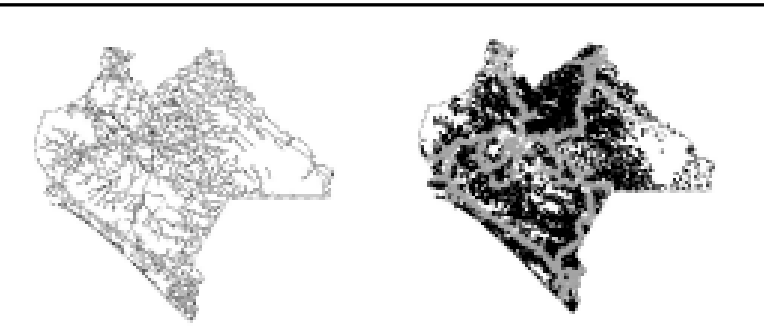

Figura 5. Ejemplo de selección por proximidad. Se seleccionaron aquellas localidades próximas a las carreteras pavimentadas que existían en los años setenta. 
Finalmente, existe una familia de análisis orientada a los sistemas de redes. Un SIG puede simular flujos a lo largo de una red de líneas que lo constituye. Es posible asignar valores dentro de la red, como dirección y resistencia, que bien pueden usarse para realizar análisis de sistemas viales o para evaluar redes hidrológicas.

Los métodos que mostramos aquí no son en absoluto los únicos posibles, pero nos dan una idea de lo que se puede lograr. Con un SIG se pueden elaborar consultas basadas en atributos de entidad, ubicación o de la combinación de ambos rasgos, pero también se generan nuevas bases de datos a partir de las consultas.

\section{Representación de los resultados}

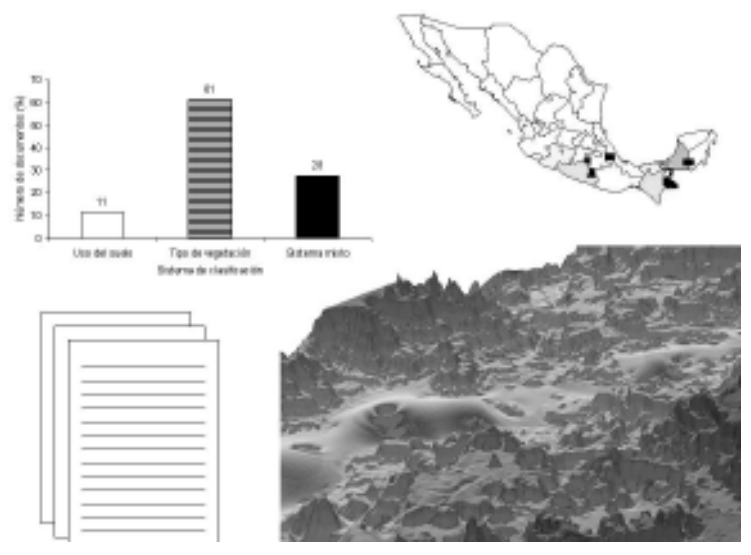

En primera instancia, la publicación de los resultados de un SIG deben ser mapas, sin embargo, no siempre es el caso. Antes habíamos dicho que una imagen dice más que mil palabras, pero a veces una cifra puede decirnos más que muchos dibujos. La generación de tablas para describir, por ejemplo, las superficies que corresponden a los diferentes tipos de vegetación puede ser más útil que ver los distintos manchones en el mapa. En el mismo sentido, un histograma de frecuencias nos daría una mejor idea de la distribución de edades de la población que una tabla. Cada caso requiere distintas acciones y la versatilidad de los SIG ha podido responder a tales requerimientos.

A veces los resultados son nuevas capas de información que pueden distribuirse en formato digital. Esto no quiere decir que los mapas no seguirán existiendo, la diferencia es que ahora es más fácil contar con mapas hechos "a la medida", y no me refiero al tamaño o escala de impresión, sino a la posibilidad de elaborarlos con la información y el formato necesarios.

En últimas fechas en el LAIGE se ha notado una tendencia en que quien solicita información ya no re- quiere un mapa impreso, pues es suficiente que se lleve un archivo JPEG de la composición cartográfica que requiere, porque esto le facilitará incluir la información en el documento donde está reportando la información.

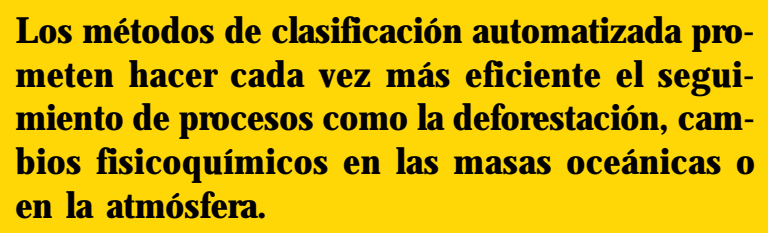

\section{¿Q $Q$ ué podemos esperar?}

Si hace 10 años alguien me hubiera contado que en 2002 tendríamos discos duros de 60 gigabytes, hubiera tenido que poner cara de inteligente mientras pensaba a cuantos malditos kilobytes corresponde un gibabyte. El desarrollo tecnológico sigue aumentando de velocidad cada día. Sin embargo, creo que en adelante los adelantos en los SIG van a estar relacionados con el desarrollo del análisis geográfico, y es que sin el segundo no existirían los primeros. Considero que mientras una parte del desarrollo en los SIG se va a dirigir al manejo de información obtenida de sensores remotos hiperespectrales (imágenes de satélite con muchas bandas) gran parte de la investigación se enfocará en nuevas aproximaciones conceptuales que permitan, por ejemplo, trabajar con límites difusos, es decir, aquellos que no pueden definirse con una línea. Los avances en el conocimiento sobre redes neuronales y en el manejo de la estadística espacial van a convertirse en pilares de nuevas formas de aproximarse a la realidad, con métodos pero también con actitudes más creativos, abiertos y frescos.

\section{Literatura citada:}

Berry, J.K. 1993. Beyond mapping: conoepts, algorithms, and issues in G IS. G IS World Books. Fort Collins.

Bocco, G . 1999. A puntes del C urso de A nálisis G eográfico. Posgrado en Ciencias Biológicas. UNAM.

Cambrezy, L., 1997. Visión del espacio y representación cartográfica. En: O. Hoffman y. F. I. Salmerón (coord.). N ueve estudios sobre el espacio. Centro de Investigaciones y Estudios Superiores en Antropología Social. Ediciones de la Casa Chata, México.

D antas-Bitencourt, M. y V. Regina-Pivello. 1998. Sig e sensoramiento remoto orbital aux iliando 0 zonamiento eoológico. Investigaciones G eográficas Boletín, 36: 35-43.

March-M. I. 1993. E l sistema de información geográfica del CIE S en la evaluación y planificación regional. Manuscrito.

NCG IA. 1990. Core Curriculum. Introduction to G IS. Vol 1. National Center for G eographic Information and Analysis/ University of California. Santa Bárbara. 